\title{
Special issue on soft computing techniques in cluster and grid computing systems
}

\author{
Bernabé Dorronsoro • Sergio Nesmachnow
}

Received: 15 November 2013 / Accepted: 19 November 2013 / Published online: 12 February 2014

(C) Springer Science+Business Media New York 2014

The resolution of complex problems requires the use of advanced approximate algorithms and huge computational resources to find accurate solutions in reasonable time. Computing clusters, as well as GPGPUs, grids, and cloud computing infrastructures arise as appropriate innovative computing platforms to implement and execute such algorithms and find accurate solutions to complex problems arising in many application areas.

This special issue contains extended versions of the best papers presented in HPCLATAM-2012 and SCCG-2012 conferences, as well as other novel contributions. In total, 27 papers were submitted to the special issue, from which only 10 were finally accepted for publication. These are outstanding contributions proposing novel designs of highly efficient parallel algorithms to solve complex problems, or dealing with salient problems in the fields of grid and cloud computing.

In the first paper of this compilation, "Approximating Static List Schedules in Dynamic Multithreaded Applications", Camargo et al. propose in their contribution the use of list algorithms to optimally schedule algorithms in a multi-thread environment. The difficulty of the approach deals with the fact that a dynamic environment is considered, and no assumptions are assumed to be known about the programs. A novel algorithm is presented to build a directed cyclic graph representing a multithread program from a data flow description, in form of a directed acyclic graph. Several

B. Dorronsoro $(\varangle)$

University of Lille 1, Lille, France

e-mail: bernabe.dorronsoro_diaz@inria.fr

S. Nesmachnow

Universidad de la República, Montevideo, Uruguay

e-mail: sergion@fing.edu.uy list scheduling algorithms are proposed and analyzed, even comparing them with the static case.

In the work "Multi-Objective Workflow Scheduling in Amazon EC2" by Durillo and Radu, the problem of scheduling scientific applications in the cloud is tackled from a multi-objective point of view. The MOHEFT algorithm studied provides a variety of trade-off optimal solutions that allow the user to choose a solution that better fits his/her needs and proprieties. The obtained results are compared versus two other state-of-the-art techniques, outperforming both of them in most cases.

In "The Sandpile Scheduler: How self-organized criticality may lead to dynamic load-balancing", Jimenez-Laredo et al. present a novel decentralized and distributed algorithm for dynamic load balance of bag-of-tasks in large datacenters. Unlike other schedulers, in addition to its decentralized nature, no knowledge is considered to be known on the tasks (as their duration). The algorithm, which is completely scalable, is shown to quickly find near optimal solutions for the studied problem instances.

A novel parallel algorithm is proposed by Mezmaz et al. in "Solving the Three Dimensional Quadratic Assignment Problem on a Computational Grid" to find exact solutions to a well-known NP-Hard problem. The authors propose a new parallel design of the well-known branch and bound algorithm by efficiently dealing with dynamic load balancing and fault tolerance of the algorithm in the grid. The optimal solution of Nug15 instance of the considered problem was found in this work for the first time.

The paper "Enhancing Distributed EAs by a Proactive Strategy" by Salto et al. presents an improvement for distributed evolutionary algorithms thanks to a new strategy for adaptive migration and mutation schemes in order to keep an appropriate diversity level in the different subpopulations. Subpopulations take decisions about migration policy and 
mutation based on local information, and they transmit it to their neighboring ones. The novel approach is shown to outperform other traditional genetic algorithms on the wellknown NK-landscape problem.

In "A general purpose parallel block structured open source incompressible flow solver", G. Usera et al. introduce caffa3d.MBRi an open source MPI-based parallel framework for solving computational fluid dynamic problems. The framework has several features, including the semiautomatic handling of complex geometries, the combination of body fitted blocks of grids, and high level communication tasks for the parallel execution. The solver is evaluated for real world applications from diverse scientific domains (bioengineering, climate forecasting, etc.)

The paper "GPGPU implementation of the BFECC algorithm for pure advection equations" by S. Costarelli et al. presents a parallel implementation in GPU for the Back and Forth Error Compensation and Correction algorithm to solve transient pure advection equations. The proposed method shows a good performance when compared against a previous implementation of the explicit version of the solver over 2D and 3D classical benchmark problems.

Cao et al., propose using both the CPU and GPU computing capabilities to develop a high-order double-precision solver for 3D compressible viscous flow problems in the article "CPU/GPU Computing for A Multi-block Structured Grid Based High-Order Flow Solver on A Large Heterogeneous System". The authors introduce a workload balancing model for distributing CPU/GPU workloads, and two strategies to overlap computations and communications. Large speedup values are reported for both the single-GPU and the multi-GPU versions, which indeed provide a significant advantage in performance.

The approach described in the paper "PSysCal: Parallel tool for Calibration of Ecosystem Models" by A. Agraz et al. is a novel methodology that is able to greatly improve the calibration quality of an ecosystem model. The proposed parallel heuristic algorithm significantly reduces the computational cost, being practical for solving large model instances even with limited computational resources.

Finally, a parallel heuristic method for obtaining a concrete approximants for a given function is presented in the article "Heuristic Quadratic Approximation for the Universality Theorem", by G. Hernandez et al. The authors tackle a computationally highly challenging problem from mathematics, which is widely applied in engineering applications, showing that the parallel version of the proposed algorithm is able to compute accurate results in efficient execution times.

We expect that the reader will find this special issue very interesting for his/her research activities. We compiled in it some relevant recent works in the field of high performance parallel computing.

B. Dorronsoro \& S. Nesmachnow 